\title{
Uso de Tromboaspiração em Angioplastia Coronária Primária no Mundo Real. Registro Multicêntrico Argentino de Infarto Agudo do Miocárdio com Supradesnivelamento do Segmento ST
}

\author{
Gerardo Nau' ${ }^{1}$, Fernando Cura ${ }^{1}$, Mariano Albertal ${ }^{1}$, Daniel Berrocal ${ }^{2}$, Oscar Mendiz³ , \\ Anibal Damonte ${ }^{4}$, Lucio Padilla ${ }^{1}$, Jorge A. Belardi ${ }^{1}$
}

\section{RESUMO}

Introdução: $\mathrm{O}$ uso de tromboaspiração manual demonstrou melhorar a reperfusão miocárdica durante a intervenção percutânea primária. Entretanto, a população adequada para esse tratamento ainda não foi bem estabelecida. Este estudo teve como objetivo determinar a taxa de utilização e a seleção de pacientes para tromboaspiração manual durante intervenção percutânea primária. Método: No período de julho de 2008 a fevereiro de 2009, incluímos 183 pacientes com intervenção percutânea primária de oito centros argentinos em um registro prospectivo de infarto do miocárdio com supradesnivelamento do segmento ST (IAMCSST). As características clínicas, angiográficas e do procedimento e os desfechos clínicos foram comparados entre os pacientes tratados com e sem tromboaspiração manual. Resultados: A tromboaspiração manual foi utilizada em 20,8\% dos pacientes. As características clínicas basais foram, em geral, semelhantes entre os pacientes tratados com e sem tromboaspiração manual. Entretanto, os pacientes tratados com tromboaspiração manual tinham pior função renal pré-procedimento e maior somatória de supradesnivelamento do segmento ST basal $(12,2 \pm 7,7 \mathrm{~mm}$ vs. $10,2 \pm 8,6 \mathrm{~mm} ; \mathrm{P}=0,06)$. $\mathrm{O}$ grupo com tromboaspiração manual apresentou maior proporção de pacientes com trombo graus $4-5(86,8 \%$ vs. $49 \%$; $\mathrm{P}<0,01)$, maior fluxo TIMI 0 basal (tromboaspiração manual: $63,2 \%$ vs. $40,7 \%$; $P=0,03$ ), maior diâmetro do vaso $(3,46 \pm 0,5 \mathrm{~mm}$ vs. $3,12 \pm 0,5 \mathrm{~mm}$; $P=0,01)$, e maior utilização de filtro distal $(13,1 \%$ vs. $0,7 \% ; P=0,04)$ e de balão intra-aórtico $(10,5 \%$ vs. $3,4 \%$; $\mathrm{P}=0,05)$, assim como de inibidores da glicoproteína $\mathrm{Ilb} / \mathrm{Illa}$

\section{ABSTRACT}

Real World Use of Thrombus-Aspiration During Primary Angioplasty. Argentinean Multicenter Registry of ST-Elevation Myocardial Infarction

Background: Manual thrombus-aspiration has proven to improve myocardial reperfusion during primary percutaneous coronary intervention. However, the adequate population for this approach has not been well established. The aim of this study was to determine the frequency and patient selection for thrombus-aspiration during primary percutaneous coronary intervention. Method: From July 2008 to February 2009, we included 183 patients submitted to primary percutaneous coronary intervention from eight Argentinean centers in a prospective ST-segment elevation myocardial infarction (STEMI) registry. Baseline characteristics as well as clinical and angiographic outcomes were compared among patients treated with and without thrombus-aspiration. Results: Manual thrombus-aspiration was used in $20.8 \%$ of the patients. Baseline clinical characteristics were similar among patients treated with and without thrombus-aspiration. However, thrombus-aspiration treatment was associated with worse baseline renal function and greater baseline ST-segment elevation (thrombus-aspiration: $12.2 \pm 7.7 \mathrm{~mm}$ vs. $10.2 \pm 8.6 \mathrm{~mm}$; $\mathrm{P}=0.06)$. The thrombus-aspiration group had a higher rate of patients with grade $4-5$ thrombus $(86.8 \%$ vs. $49 \% ; \mathrm{P}<0.01)$ and baseline TIMI flow 0 (thrombus-aspiration $63.2 \%$ vs. $40.7 \%$; $\mathrm{P}=0.03$ ), greater vessel diameter $(3.46 \pm 0.5 \mathrm{~mm}$ vs. $3.12 \pm 0.5 \mathrm{~mm}$; $\mathrm{P}=0.01)$, use of distal filter $(13.1 \%$ vs. $0.7 \% ; P=0.04)$

\footnotetext{
1 Instituto Cardiovascular de Buenos Aires (ICBA) - Buenos Aires, Argentina.

2 Hospital Italiano de Buenos Aires - Buenos Aires, Argentina.

3 Hospital Universitário de la Fundación Favaloro - Buenos Aires, Argentina.

${ }^{4}$ Instituto Cardiovascular de Rosario - Rosario, Argentina.

Correspondência: Gerardo Nau. Tres Sargentos, 2046 - Martinez Buenos Aires, Argentina.

E-mail: g_nau@yahoo.com

Recebido em: 28/5/2009 - Aceito em: 20/9/2009
} 
Nau G, et al. Uso de Tromboaspiração em Angioplastia Coronária Primária no Mundo Real. Registro Multicêntrico Argentino de Infarto Agudo do Miocárdio com Supradesnivelamento do Segmento ST. Rev Bras Cardiol Invas. 2009;17(3):340-5.

$(39,5 \%$ vs. $14,5 \% ; P=0,04)$. A taxa de resgate de material aterotrombótico macroscópico foi de 76,3\%. Embora o grupo com tromboaspiração manual apresentasse pico mais elevado de creatina fosfoquinase (tromboaspiração manual: $3.195,9 \pm 2.598 \mathrm{Ul} / \mathrm{ml}$ vs. $1.757,6 \pm 1.806,6 \mathrm{Ul} / \mathrm{ml}$; $\mathrm{P}=0,02)$, a porcentagem de resolução do segmento ST e o fluxo TIMI 3 final não foram diferentes entre os grupos. A taxa de eventos cardiovasculares adversos maiores no acompanhamento foi semelhante $(21,1 \%$ vs. $15,2 \% ; \mathrm{P}=0,36)$. Conclusão: $O$ emprego da tromboaspiração manual na prática diária em paciente com IAMCSST destina-se a pacientes de maior complexidade tanto clínica como angiográfica, acarretando, no final, resultados clínicos semeIhantes aos de pacientes não tratados com tromboaspiração manual.

DESCRITORES: Trombectomia. Angioplastia transluminal percutânea coronária. Infarto do miocárdio/terapia. Trombose coronária.

A intervenção coronária percutânea primária é o tratamento preferencial para obter reperfusão miocárdica em infarto agudo do miocárdio com supradesnivelamento do segmento $\mathrm{ST}^{1}$. O stent, inicialmente contraindicado na presença de trombo intracoronário, tem demonstrado bons resultados nesse tipo de lesão, ao alcançar maior diâmetro luminal no final do procedimento e resolver dissecções residuais, fatores que predispõem à isquemia e à reoclusão do vaso $^{2}$. A presença do trombo intracoronário pode, no entanto, levar à embolização distal durante intervenção coronária percutânea, e a obstrução microvascular resultante reduz a perfusão miocárdica, estando associada a maior perda de músculo cardíaco, menor recuperação da função ventricular e maior mortalidade ${ }^{3,4}$.

Vários sistemas para trombectomia intracoronária, que diferem em desenho, mecanismos e manuseio, foram introduzidos na prática clínica da intervenção coronária percutânea primária ${ }^{5,6}$. Estudos recentes sugerem que a trombectomia com aspiração manual durante a intervenção coronária percutânea primária, em pacientes com trombo intracoronário visível, pode prevenir a embolização distal, melhorando a perfusão miocárdica e reduzindo a extensão e o remodelamento do ventrículo esquerdo ${ }^{7,8}$. Entretanto, a população adequada para esse tipo de tratamento não foi bem estabelecida. Este estudo teve por objetivo determinar a taxa de utilização e a seleção de pacientes para trombectomia com aspiração manual durante a intervenção coronária percutânea primária.

\section{MÉTODO}

\section{População do estudo e procedimento}

No período de julho de 2008 a fevereiro de 2009, incluímos 183 pacientes consecutivos submetidos a intervenção coronária percutânea primária em oito cen- and use of intra-aortic balloon $(10.5 \%$ vs $3.4 \%$; $P=0.05)$, as well as glycoprotein IIb/IIla inhibitor administration $(39.5 \%$ vs. $14.5 \% ; P=0.04)$. Debris was retrieved from $76.3 \%$ of the patients. Although the thrombus-aspiration group had higher CK levels (thrombus-aspiration: $3195.9 \pm$ $2598 \mathrm{UI} / \mathrm{ml}$ vs. $1757.6 \pm 1806.6 \mathrm{UI} / \mathrm{ml} ; \mathrm{P}=0.02)$, the percentage of ST-segment resolution and final TIMI 3 flow did not differ between groups. The follow-up rate of major cardiovascular events was similar for both groups (thrombusaspiration: $21.1 \%$ vs. $15.2 \% ; \mathrm{P}=0.36)$. Conclusion: Routine thrombus-aspiration in patients with STEMI is used in patients with higher clinical and angiographic complexity, leading to similar overall clinical results as those not treated with thrombus-aspiration.

DESCRIPTORS: Thrombectomy. Angioplasty, transluminal, percutaneous coronary. Myocardial infarction/therapy. Coronary thrombosis.

tros argentinos em registro prospectivo e contemporâneo de infarto agudo do miocárdio com supradesnivelamento do segmento ST. Os pacientes incluídos apresentavam dor torácica isquêmica por período superior a 30 minutos e supradesnivelamento do segmento $\mathrm{ST} \geq 2 \mathrm{~mm}$ em pelo menos duas derivações eletrocardiográficas contíguas, nas primeiras 24 horas de evolução.

Todos os pacientes foram pré-tratados com $300 \mathrm{mg}$ de aspirina por via oral e recomendou-se $600 \mathrm{mg}$ de clopidogrel como dose de ataque antes da angioplastia. Dependendo do centro, usou-se heparina intravenosa ou bivalirrudina para manter tempo de coagulação ativada superior a 250 segundos. A administração de antagonistas de glicoproteína Ilb/Illa ficou a critério do operador. A trombectomia com aspiração manual era recomendada a pacientes com infarto agudo do miocárdio com supradesnivelamento do segmento ST e lesão com grande carga de trombos (graus 4-5, fluxo coronário TIMI 0-1 após o cruzamento da lesão com a guia), localizada em segmento coronário proximal, em vasos com diâmetro superior a 2,5 mm.

A angiografia coronária foi analisada quanto a características da lesão, presença de trombo e embolização distal, antes e após a intervenção coronária percutânea primária. A carga de trombo no local da lesão foi graduada de 0 a 5, conforme a escala TIMI de trombo ${ }^{9}$. Além disso, estimou-se visualmente o grau de fluxo coronário pela classificação do TIMI: o slowflow (fluxo TIMI com redução de grau 3 para 2 durante o procedimento) e o no-reflow (fluxo TIMI com redução de grau 3 ou 2 para 0 ou 1 durante o procedimento $)^{10,11}$. Todos os pacientes realizaram eletrocardiograma de 12 derivações antes e 90 minutos após o procedimento. O supradesnivelamento do segmento ST foi medido manualmente. A resolução do 
segmento ST foi expressa como porcentual de resolução do supradesnivelamento inicial do segmento ST: porcentual $>70 \%$ foi indicativo de reperfusão miocárdica bem-sucedida 90 minutos após o procedimento índice; porcentual de 30\% a $70 \%$ foi considerado reperfusão parcial; e porcentual $<30 \%$ foi considerado não-reperfusão ${ }^{12}$.

O acompanhamento foi realizado de forma prospectiva por um mês. A partir dos registros hospitalares e de entrevistas por telefone, avaliamos a ocorrência de eventos cardiovasculares adversos maiores (morte cardíaca, infarto do miocárdio ou revascularização de vaso-alvo). Também avaliamos a incidência de trombose do stent indicada como definitiva, provável e possível, conforme a definição do Academic Research Consortium ${ }^{13}$.

\section{Análise estatística}

As variáveis categóricas foram expressas como números e porcentuais e analisadas pelo teste de qui-quadrado ou pelo teste exato de Fisher, conforme indicado. As variáveis contínuas foram expressas como média \pm desvio padrão e analisadas pelo teste $t$ de Student. As diferenças foram consideradas significativas para valores de $P \leq 0,05$. A análise estatística foi feita com o programa SPSS (Statistical Package for the Social Sciences).

\section{RESULTADOS}

\section{Características basais}

Este registro incluiu 183 pacientes consecutivos com infarto agudo do miocárdio com supradesnivelamento do segmento ST, sendo realizada tromboaspiração manual em 38 (20,8\%) indivíduos. As características clínicas basais desses pacientes eram semelhantes às de pacientes tratados sem tromboaspiração manual. Entretanto, o tratamento com tromboaspiração manual foi associado a menor taxa de filtração glomerular basal $(68,4 \pm 24,2 \mathrm{ml} / \mathrm{min}$ vs. $77,3 \pm 27,3 \mathrm{ml} / \mathrm{min} ; \mathrm{P}=0,03$ ) e maior território miocárdico isquêmico, traduzido pela maior somatória de supradesnivelamento do segmento ST basal (12,2 \pm 7,7 mm vs.10,2 $\pm 8,6 \mathrm{~mm} ; \mathrm{P}=0,06$ ) (Tabela 1). No grupo com tromboaspiração manual, os inibidores de glicoproteína IIb/IIla (39,5\% vs. 14,5\%; P =0,04) e a contrapulsação com balão intra-aórtico (10,5\% vs. $3,4 \% ; P=0,05)$ foram utilizados com maior frequência (Tabela 2).

\section{Achados angiográficos e do procedimento}

A artéria descendente anterior foi tratada em porcentual semelhante entre os grupos (34,2\% vs. 45,5\%; $\mathrm{P}=0,17)$ e a tromboaspiração manual foi realizada

TABELA 1

Características clínicas e angiográficas

\begin{tabular}{|c|c|c|c|}
\hline & Tromboaspiração manual $(\mathbf{n}=38)$ & ICP convencional $(n=145)$ & $\mathbf{P}$ \\
\hline Idade, anos (média \pm DP) & $60,1 \pm 13,7$ & $62,2 \pm 13,3$ & 0,12 \\
\hline Sexo masculino (\%) & $33(86,8)$ & $113(77,9)$ & 0,09 \\
\hline \multicolumn{4}{|l|}{ Fatores de risco } \\
\hline Hipertensão (\%) & $22(57,9)$ & $87(60,0)$ & 0,15 \\
\hline Diabetes melito (\%) & $6(15,8)$ & $23(15,9)$ & 0,42 \\
\hline Tabagismo (\%) & $18(47,4)$ & $48(33,1)$ & 0,09 \\
\hline Hipercolesterolemia (\%) & $21(55,3)$ & $78(53,8)$ & 0,15 \\
\hline Infarto do miocárdio prévio (\%) & $6(15,8)$ & $16(11,0)$ & 0,09 \\
\hline Revascularização prévia (\%) & $4(10,5)$ & $18(12,4)$ & 0,16 \\
\hline Killip > I $(\%)$ & $14(36,8)$ & $6(24,8)$ & 0,12 \\
\hline Filtração glomerular, ml/min \pm DP & $68,4 \pm 24,2$ & $77,3 \pm 27,3$ & 0,03 \\
\hline$\sum$ supra de $\mathrm{ST}, \mathrm{mm} \pm \mathrm{DP}$ & $12,2 \pm 7,7$ & $10,2 \pm 8,6$ & 0,06 \\
\hline Resolução ST > 70\% (\%) & $16(42,1)$ & $81(55,9)$ & 0,40 \\
\hline Pico de creatina quinase, $\mathrm{UI} / \mathrm{ml} \pm \mathrm{DP}$ & $3.195,9 \pm 2.598$ & $1.757,6 \pm 1.806,6$ & 0,02 \\
\hline \multicolumn{4}{|l|}{ Características angiográficas } \\
\hline Doença multiarterial (\%) & $17(44,7)$ & $73(50,3)$ & 0,51 \\
\hline Artéria descendente anterior (\%) & $13(34,2)$ & $66(45,5)$ & 0,17 \\
\hline Diâmetro de referência do vaso, $\mathrm{mm} \pm \mathrm{DP}$ & $3,46 \pm 0,5$ & $3,12 \pm 0,5$ & 0,01 \\
\hline Calcificação grave (\%) & $3(7,9)$ & $19(13,1)$ & 0,05 \\
\hline Tortuosidade grave (\%) & $1(2,6)$ & $8(5,5)$ & 0,05 \\
\hline Trombo graus 4-5 (\%) & $33(86,8)$ & $71(49)$ & $<0,01$ \\
\hline
\end{tabular}

$\mathrm{DP}=$ desvio padrão; ICP = intervenção coronária percutânea; $\mathrm{n}$ = número de pacientes. 
Nau G, et al. Uso de Tromboaspiração em Angioplastia Coronária Primária no Mundo Real. Registro Multicêntrico Argentino de Infarto Agudo do Miocárdio com Supradesnivelamento do Segmento ST. Rev Bras Cardiol Invas. 2009;17(3):340-5.

TABELA 2

Características relacionadas ao procedimento

\begin{tabular}{|c|c|c|c|}
\hline & Tromboaspiração manual $(\mathbf{n}=38)$ & ICP convencional $(n=145)$ & $\mathbf{P}$ \\
\hline ICP em horário regular de atendimento (\%) & $18(47,4)$ & $72(49,7)$ & 0,45 \\
\hline Tempo sintoma-balão, minutos \pm DP & $240 \pm 163$ & $311 \pm 306$ & 0,09 \\
\hline Clopidogrel 600 mg (\%) & $34(89,5)$ & $107(73,7)$ & 0,56 \\
\hline Uso de inibidores de glicoproteína IIb/IIla (\%) & $15(39,5)$ & $21(14,5)$ & 0,04 \\
\hline Uso de BIA (\%) & $4(10,5)$ & $5(3,4)$ & 0,05 \\
\hline Uso de filtro distal (\%) & $5(13,1)$ & $1(0,7)$ & 0,04 \\
\hline Trombo aspirado (\%) & $29(76,3)$ & - & NA \\
\hline ICP em múltiplos vasos & $1(2,6)$ & $15(10,3)$ & 0,08 \\
\hline Número de stents/paciente & $1,32 \pm 0,7$ & $1,37 \pm 0,5$ & 0,45 \\
\hline Fluxo TIMI 0 pré-trombectomia (\%) & $24(63,2)$ & $59(40,7)$ & 0,03 \\
\hline Fluxo TIMI 3 final (\%) & $26(68,4)$ & $89(61,4)$ & 0,08 \\
\hline No reflow $(\%)$ & $7(18,4)$ & $13(9)$ & 0,14 \\
\hline
\end{tabular}

BIA = balão intra-aórtico; $D P=$ desvio padrão; $I C P$ = intervenção coronária percutânea; $\mathrm{n}=$ número de pacientes; $N A=$ não avaliado.

em vasos de maior diâmetro $(3,46 \pm 0,5 \mathrm{~mm}$ vs. 3,12 \pm $0,4 \mathrm{~mm} ; \mathrm{P}=0,01)$, com maior quantidade de trombos (86,8\% vs. 49\%; P<0,01), menor calcificação $(7,9 \%$ vs. $13,1 \% ; P=0,05)$ e tortuosidade $(2,6 \%$ vs. $5,5 \%$; $\mathrm{P}=0,05)$ (Tabela 1).

Além disso, os pacientes tratados com dispositivo de aspiração mostraram incidência significativamente maior de fluxo TIMI 0 basal (63,2\% vs. 40,7\%; $P=0,03)$ e tendência a maior fluxo TIMI 3 ao final do procedimento $(68,4 \%$ vs. $61,4 \% ; P=0,08)$. Foi identificada taxa de retirada de material aterotrombótico macroscópico de 76,3\% imediatamente após a tromboaspiração (Tabela 2). Após trombectomia manual não foi observada complicação relacionada ao uso da técnica.

\section{Resultados clínicos}

Apesar do maior pico de creatina fosfoquinase $(3.195,9 \pm 2.598 \mathrm{UI} / \mathrm{ml}$ vs. $1.757,6 \pm 1.806,6 \mathrm{UI} / \mathrm{ml}$; $\mathrm{P}=0,02)$, a resolução do segmento $\mathrm{ST}>70 \%$ foi semelhante nos dois grupos (Tabela 1 e Figura 1). Ecocardiograma foi realizado durante a hospitalização e demonstrou não haver diferença significativa na fração de ejeção do ventrículo esquerdo entre os grupos $(43,7 \pm 3,6 \%$ vs. $49,8 \pm 13 \% ; P=0,09)$. Não foram observadas diferenças significativas quanto à incidência de eventos cardiovasculares adversos maiores durante a hospitalização e no acompanhamento aos 30 dias. Todas as mortes cardíacas ocorreram durante a hospitalização. No grupo submetido a tromboaspiração manual, observamos dois casos de trombose do stent, estatisticamente semelhante à intervenção coronária percutânea convencional, e um desses pacientes faleceu (Tabela 3).

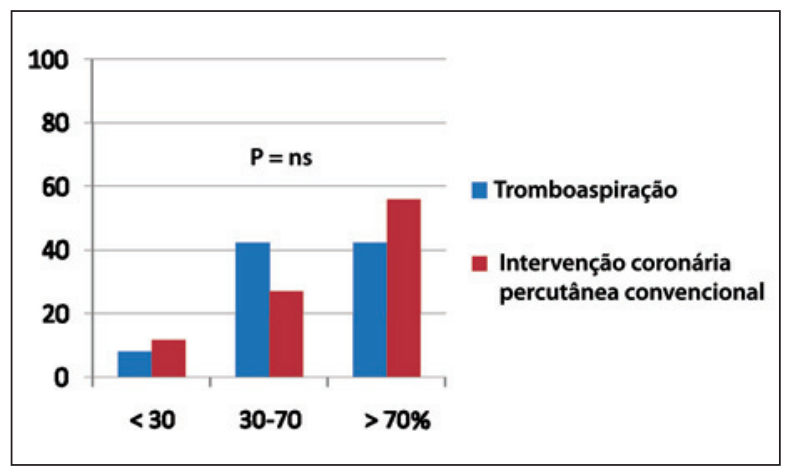

Figura 1 - Resolução ST aos 90 minutos. ns = não-significante.

\section{DISCUSSÃO}

A intervenção coronária percutânea primária é atualmente a estratégia padrão no tratamento do infarto agudo do miocárdio com supradesnivelamento do segmento ST, capaz de reduzir a morbidade e a mortalidade. A reperfusão tecidual ideal é um preditor positivo dos resultados de longo prazo e trombos visíveis pela angiografia estão associados a piores resultados agudos e tardios ${ }^{14,15}$. O principal achado deste estudo foi que a aspiração de trombo antes do implante de stent durante intervenção coronária percutânea primária foi realizada em um subgrupo clínico de mais alto risco (maior supradesnivelamento do segmento ST e maior necessidade de inibidores de glicoproteína IIb/IIla e de balão intra-aórtico) em associação com um padrão angiográfico com piores características (fluxo TIMI grau 0 e maior quantidade de trombos).

Nosso estudo analisa o papel da tromboaspiração manual em uma população representativa e contempo- 
Nau G, et al. Uso de Tromboaspiração em Angioplastia Coronária Primária no Mundo Real. Registro Multicêntrico Argentino de Infarto Agudo do Miocárdio com Supradesnivelamento do Segmento ST. Rev Bras Cardiol Invas. 2009;17(3):340-5.

TABELA 3

Resultados clínicos em 30 dias

\begin{tabular}{lccc}
\hline & Tromboaspiraçãomanual $(\mathbf{n}=\mathbf{3 8})$ & ICP convencional $(\mathbf{n}=\mathbf{1 4 5})$ & $\mathbf{P}$ \\
\hline Eventos cardíacos adversosmaiores $(\%)$ & $8(21,1)$ & $22(15,2)$ & 0,36 \\
Morte cardíaca $(\%)$ & $3(7,9)$ & $11(7,6)$ & 0,70 \\
Reinfarto $(\%)$ & $2(5,3)$ & $1(0,7)$ & 0,12 \\
Revascularização dovaso-alvo (\%) & $2(5,3)$ & $1(0,7)$ & 0,08 \\
Trombose do stent definitiva/provável (\%) & $2(5,3)$ & $1(0,7)$ & 0,09 \\
Hemorragia grave $(\%)$ & 0 & $2(1,4)$ & 0,45 \\
\hline
\end{tabular}

rânea com infarto agudo do miocárdio com supradesnivelamento do segmento ST. Korn et al. ${ }^{16}$ avaliaram o benefício da trombectomia adjunta à intervenção coronária percutânea e demonstraram não haver benefício claro para qualquer grupo de pacientes. Entretanto, o estudo incluiu todos os tipos de síndromes coronárias agudas, dificultando a avaliação do benefício real nos pacientes com diferentes fisiopatologias e prognósticos.

Em comparação com o grupo tratado com intervenção coronária percutânea primária convencional, o grupo com aspiração manual apresentou reperfusão do miocárdio satisfatória, documentada por melhora do grau de fluxo coronário TIMI, por resolução do supradesnivelamento do segmento ST, e pela não ocorrência de aumento do fenômeno de no-reflow, apesar do maior risco desses pacientes.

As diferenças dos resultados encontrados na literatura podem estar relacionadas ao dispositivo utilizado, já que os estudos envolvendo a aspiração manual têm demonstrado efeitos mais favoráveis relacionados à perfusão do miocárdio ${ }^{5}$. Os dispositivos manuais apresentam preparo e uso fáceis. Uma vez que a equipe tenha adquirido experiência suficiente, a tromboaspiração manual pode ser utilizada em situações clínicas complexas que requerem ação rápida e eficiente. Entretanto, em nosso estudo, nos casos em que a anatomia coronária apresentava intensa tortuosidade, calcificações ou segmentos distais tratados, a tromboaspiração manual não foi escolhida pelo operador. Devemos selecionar os pacientes cuidadosamente para evitar as complicações relacionadas à técnica, proporcionando maior fluxo epicárdico pós-trombectomia e perfusão microvascular adequada após a colocação do stent.

Quando a anatomia coronária é adequada, os dispositivos de aspiração são de fácil uso (indicado pela duração semelhante do tempo porta-balão como grupo controle) e seguros (sem dissecções limitadoras de fluxo ou outras complicações relacionadas ao dispositivo durante o procedimento). Ao contrário dos estudos anteriores, não observamos perfuração ou dissecções limitadoras de fluxo ou outras complicações relacionadas ao dispositivo durante o procedimento ${ }^{17}$.
A taxa de retirada de material aterotrombótico foi elevada $(76,3 \%)$ e semelhante aos estudos anteriores em pacientes tratados com infarto agudo do miocárdio com supradesnivelamento do segmento $\mathrm{ST}^{18,19}$. Não identificamos material aterotrombótico em 23,7\% dos pacientes submetidos a aspiração. Isso pode ser decorrente de vários mecanismos, como tratamento bemsucedido com antitrombóticos ou fibrinolíticos, embolização antes ou durante intervenção coronária percutânea ou, ainda, coágulos muito friáveis que se desintegram à aspiração.

Embora os pacientes submetidos a tromboaspiração manual pudessem ter resultado potencialmente pior, as taxas de mortalidade de 30 dias, reinfarto e insuficiência cardíaca foram semelhantes entre os grupos. Além disso, não observamos diferença significativa na fração de ejeção do ventrículo esquerdo.

A aspiração manual de trombos pode ser realizada em pacientes selecionados com infarto agudo do miocárdio com supradesnivelamento do segmento ST, melhorando, potencialmente, a reperfusão miocárdica e os eventos clínicos em comparação com a intervenção coronária percutânea convencional.

O presente estudo apresenta algumas limitações, dentre elas o fato de ser um estudo observacional, não permitindo comparação direta dos resultados do uso de trombectomia com a reperfusão convencional. O objetivo deste estudo foi descrever a aplicabilidade e os resultados do uso de trombectomia na prática diária dos laboratórios intervencionistas. Estudos posteriores provavelmente produzirão os dados necessários para confirmar esse resultado.

\section{CONCLUSÃO}

A tromboaspiração manual durante intervenção coronária percutânea primária foi usada preferencialmente em pacientes de alto risco e em casos com carga elevada do trombo. O uso do dispositivo nesta população proporcionou resultados, em geral, semeIhantes aos dos pacientes de menor risco não tratados com tromboaspiração manual. A tromboaspiração manual mostrou-se segura e benéfica em um subgrupo que usualmente apresenta resultados desfavoráveis sem tratamento adequado. 
Nau G, et al. Uso de Tromboaspiração em Angioplastia Coronária Primária no Mundo Real. Registro Multicêntrico Argentino de Infarto Agudo do Miocárdio com Supradesnivelamento do Segmento ST. Rev Bras Cardiol Invas. 2009;17(3):340-5.

\section{CONFLITO DE INTERESSES}

Os autores declararam inexistência de conflito de interesses relacionados a este artigo.

\section{REFERÊNCIAS BIBLIOGRÁFICAS}

1. Antman EM, Anbe DT, Armstrong PW, Bates ER, Green LA, Hand $M$, et al. ACC/AHA guidelines for the management of patients with ST-elevation myocardial infarction; A report of the American College of Cardiology/American Heart Association Task Force on Practice Guidelines (Committee to Revise the 1999 Guidelines for the Management of patients with acute myocardial infarction). J Am Coll Cardiol. 2004;44(3):E1-E211.

2. Grines C, Brodie B, Griffin J, Donohue B, Samprolesi A, Constantini $C$, et al. Which primary PTCA patients may benefit from new technologies? Circulation. 1995;92 Suppl I:1146.

3. Stone GW, Peterson MA, Lansky AJ, Dangas G, Mehran R, Leon MB. Impact of normalized myocardial perfusion after successful angioplasty in acute myocardial infarction. J Am Coll Cardiol. 2002;39(4):591-7.

4. McLaughlin MG, Stone GW, Aymong E, Gardner G, Mehran R, Lansky AJ, et al. Prognostic utility of comparative methods for assessment of ST-segment resolution after primary angioplasty for acute myocardial infarction: the Controlled Abciximab and Device Investigation to Lower Late Angioplasty Complications (CADILLAC) trial. J Am Coll Cardiol. 2004;44(6):1215-23.

5. Burzotta F, Trani C, Romagnoli E, Mazzari MA, Rebuzzi AG, De Vita $M$, et al. Manual thrombus-aspiration improves myocardial reperfusion: the randomized evaluation of the effect of mechanical reduction of distal embolization by thrombus-aspiration in primary and rescue angioplasty (REMEDIA) trial. J Am Coll Cardiol. 2005;46(2):371-6.

6. Silva-Orrego P, Colombo P, Bigi R, Gregori D, Delgado A, Salvade $\mathrm{P}$, et al. Thrombus aspiration before primary angioplasty improves myocardial reperfusion in acute myocardial infarction: the DEAR-MI (Dethrombosis to Enhance Acute Reperfusion in Myocardial Infarction) study. J Am Coll Cardiol. 2006;48(8):1552-9.

7. Kondo $H$, Suzuki $T$, Fukutomi $T$, Suzuki S, Hayase $M$, Ito $S$, et al. Effects of percutaneous coronary arterial thrombectomy during acute myocardial infarction on left ventricular remodeling. Am J Cardiol. 2004;93(5):527-31.

8. Mizote I, Ueda Y, Ohtani T, Shimizu M, Takeda Y, Oka T, et al. Distal protection improved reperfusion and reduced left ventricular dysfunction in patients with acute myocardial infarction who had angioscopically defined ruptured plaque. Circulation. 2005;112(7):1001-7.
9. The TIMI IIIA Investigators. Early effects of tissue-type plasminogen activator added to conventional therapy on the culprit coronary lesion in patients presenting with ischemic cardiac pain at rest. Results of the Thrombolysis in Myocardial Ischemia. (TIMI IIIA) Trial. Circulation. 1996; 87(1):38-52.

10. TIMI Study Group. The Thrombolysis in Myocardial Infarction (TIMI) trial. Phase I findings. N Engl J Med. 1985; 312(14):932-6.

11. van't Hof AW, Liem A, Suryapranata H, Hoorntje JC, de Boer MJ, Zijlstra F. Angiographic assessment of myocardial reperfusion in patients treated with primary angioplasty for acute myocardial infarction: myocardial blush grade. Zwolle Myocardial Infarction Study Group. Circulation. 1998;97(23):2302-6.

12. van't Hof AW, Liem A, de Boer MJ, Zijlstra F. Clinical value of 12-lead electrocardiogram after successful reperfusion therapy for acute myocardial infarction. Zwolle Myocardial Infarction Study Group. Lancet.1997;350(9078):615-9.

13. Mauri L, Hsieh WH, Massaro JM, Ho KK, D'Agostino R, Cutlip DE. Stent thrombosis in randomized clinical trials of drug-eluting stents. N Engl J Med. 2007;356(10):1020-9.

14. Grines CL, Cox DA, Stone GW, Garcia E, Mattos LA, Giambartolomei $\mathrm{A}$, et al. Coronary angioplasty with or without stent implantation for acute myocardial infarction. Stent Primary Angioplasty in Myocardial Infarction Study Group. N Engl J Med. 1999;341(26):1949-56.

15. White CJ, Ramee SR, Collins TJ, Escobar AE, Karsan A, Shaw D, et al. Coronary thrombi increase PTCA risk: angioscopy as a clinical tool. Circulation. 1996;93(2):253-8.

16. Korn HV, Ohlow M, Donev S, Yu J, Huegl B, Wagner A, et al. Export aspiration system in patients with acute coronary syndrome and visible thrombus provides no substantial benefit. Catheter Cardiovasc Interv. 2007;70(1):35-42.

17. Ischinger T; X-SIZER Study Group. Thrombectomy with the X-SIZER catheter system in the coronary circulation: initial results from a multi-center study. J Invasive Cardiol. 2001; 13(2):81-8.

18. Svilaas T, Vlaar PJ, van der Horst IC, Diercks GF, de Smet $\mathrm{BJ}$, van den Heuvel AF, et al. Thrombus aspiration during primary percutaneous coronary intervention. $N$ Engl J Med. 2008;358(6):557-67.

19. Sardella G, Mancone M, Bucciarelli-Ducci C, Agati L, Scardala $\mathrm{R}$, Carbone I, et al. Thrombus aspiration during primary percutaneous coronary intervention improves myocardial reperfusion and reduces infarct size: the EXPIRA (thrombectomy with export catheter in infarct-related artery during primary percutaneous coronary intervention) prospective, randomized trial. J Am Coll Cardiol. 2009;53(4):309-15. 